\title{
CHRONIC EOSINOPHILIC PNEUMONIA WITH SUBPLEURAL CURVILINEAR SHADOW
}

\author{
Katsunori Kagohashi ${ }^{1}$, Gen Ohara ${ }^{1}$, Koichi Kurishima ${ }^{2}$, Mio Kawaguchi $^{2}$, Hidetsugu Nakayama ${ }^{4}$, \\ Hiroichi Ishikawa ${ }^{3}$,Hiroaki Satoh ${ }^{1}$
}

Department of Internal Medicine, Mito Medical Center, University of Tsukuba, Japan ${ }^{1}$; Division of Respiratory Medicine, Institute of Clinical Medicine, University of Tsukuba, Japan²; Division of Respiratory Medicine, Tsukuba Medical Center Hospital, Japan ${ }^{3}$; Department of Radiology, Tokyo Medical University, Japan ${ }^{4}$

Summary: We report a rare case of chronic eosinophilic pneumonia with subpleural curvilinear shadow. CT scan showed a patchy consolidation in the bilateral upper lungs. In addition, subpleural curvilinear shadow was found in the bilateral upper lungs. A bronchoalveolar lavage obtained from the right middle lobe showed $25 \%$ eosinophils. Although very rare, we should therefore keep in mind that patients, who have patchy consolidation with areas of subpleural curvilinear shadow in the bilateral upper lungs, may have chronic eosinophilic pneumonia.

Key words: Chronic eosinophilic pneumonia; Subpleural curvilinear shadow

\section{Introduction}

Chronic eosinophilic pneumonia (CEP) is characterized by a progressive symptomatic deterioration of more than 1 month, pulmonary infiltrates with eosinophils and the dramatic response to corticosteroid treatment $(3,4,6,9,17$, 21, 22). The exact incidence is not known. In general, CEP presents radiographically as fluffy ground-glass area of opacification with ill-defined margins located in the lung periphery, a phenomenon that has been described as the photographic negative of pulmonary edema $(3,4,6,9,17$, 21,22 ). Our patient, interestingly, presented with subpleural curvilinear shadow (SCLS) within both upper lung infiltates. As this atypical presentation has scarcely been described previously, we herein report a case with this rare finding $(6,9,18)$.

\section{Case Report}

A 64-year-old female presented with a 4-month history of non-productive cough, fever, and night sweats. Her medical history was significant for diabetic mellitus. The patient did not smoke and she was not exposed to toxins, tropical diseases and legal or illegal drugs. A chest radiograph obtained by her general practitioner was suspicious of peripheral pulmonary infiltrates. The physical examination was unremarkable. No lymphadenopathy was present. Laboratory data were remarkable for white cell count of $9.5 \times 10^{9} / \mathrm{L}$ with $23 \%$ eosinophils and an immunoglobulin E (IgE) titer of $183 \mathrm{U} / 1$. No biliary parasite was identified by sonography. Egg of parasite was not found in the stools and serological tests were negative for parasites. The chest radiograph on admission showed peripherally predominant pulmonary infiltrates, especially in the upper part of the both lungs (Fig. 1). CT scan showed a patchy consolidation

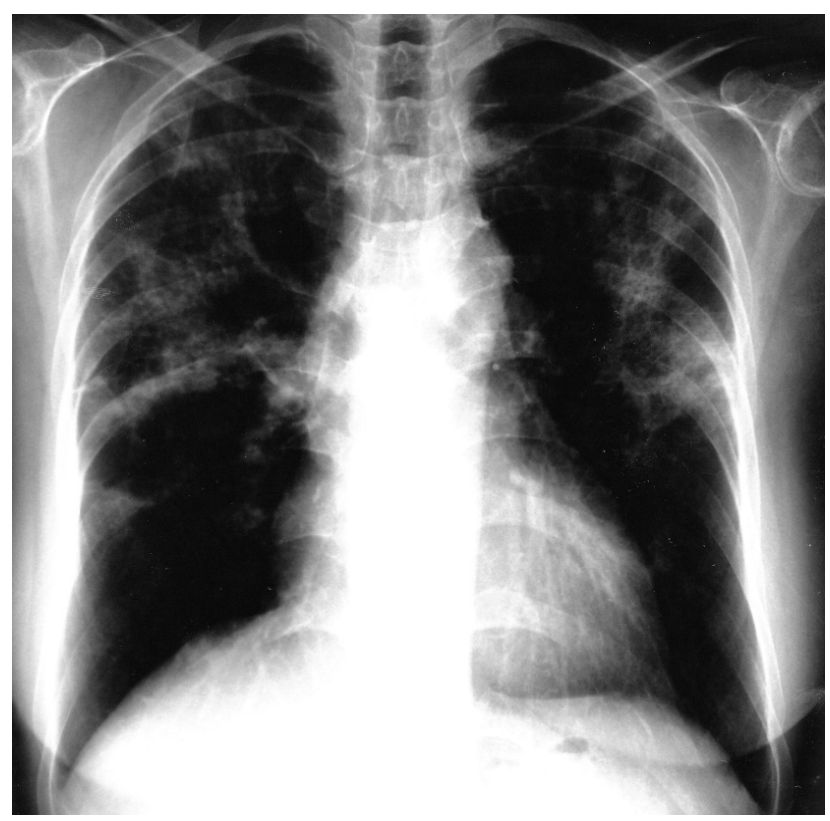

Fig. 1: The chest radiograph on admission showed peripherally predominant pulmonary infiltrates, especially in the upper part of the both lungs. 


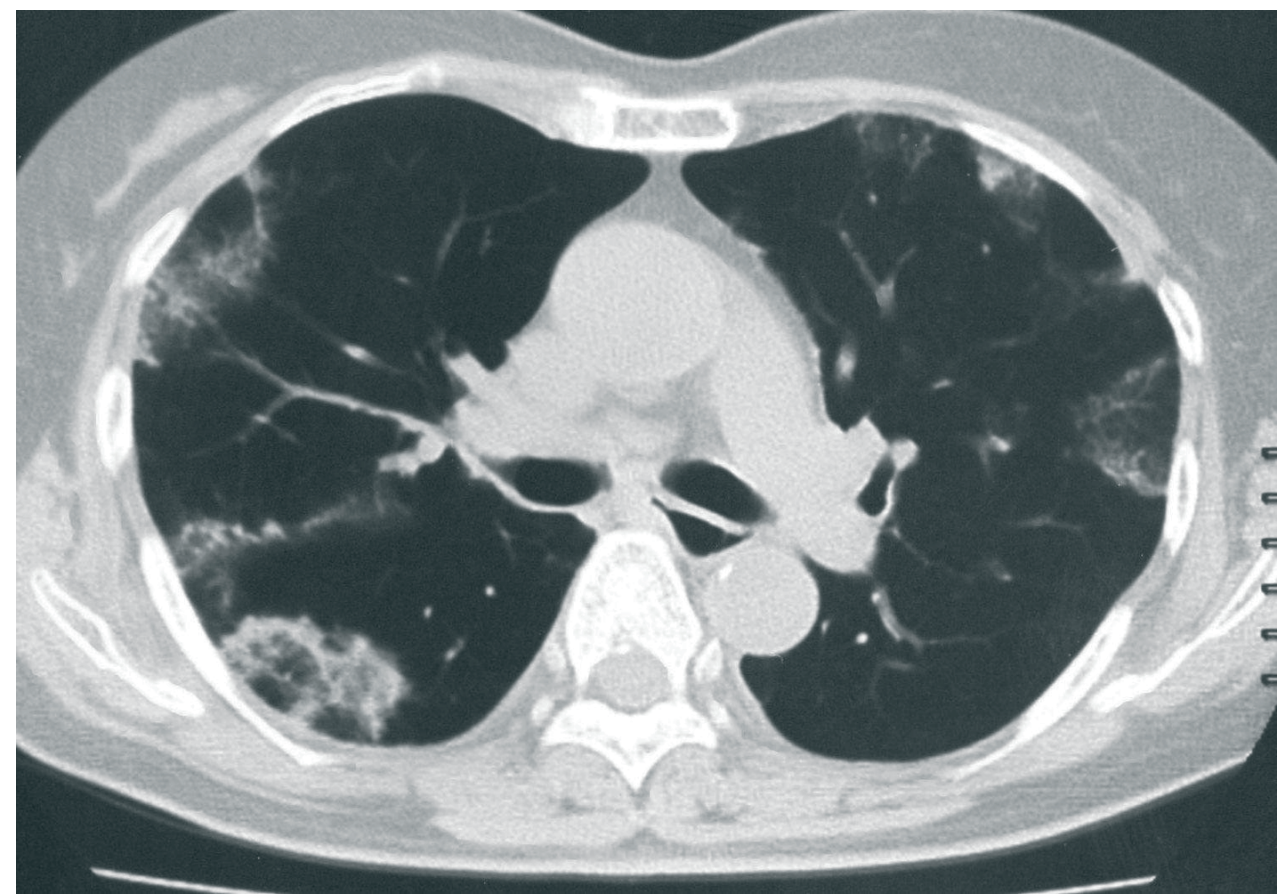

Fig. 2: CT scan on admission showed patchy consolidation in bilateral upper lobe of the lungs.

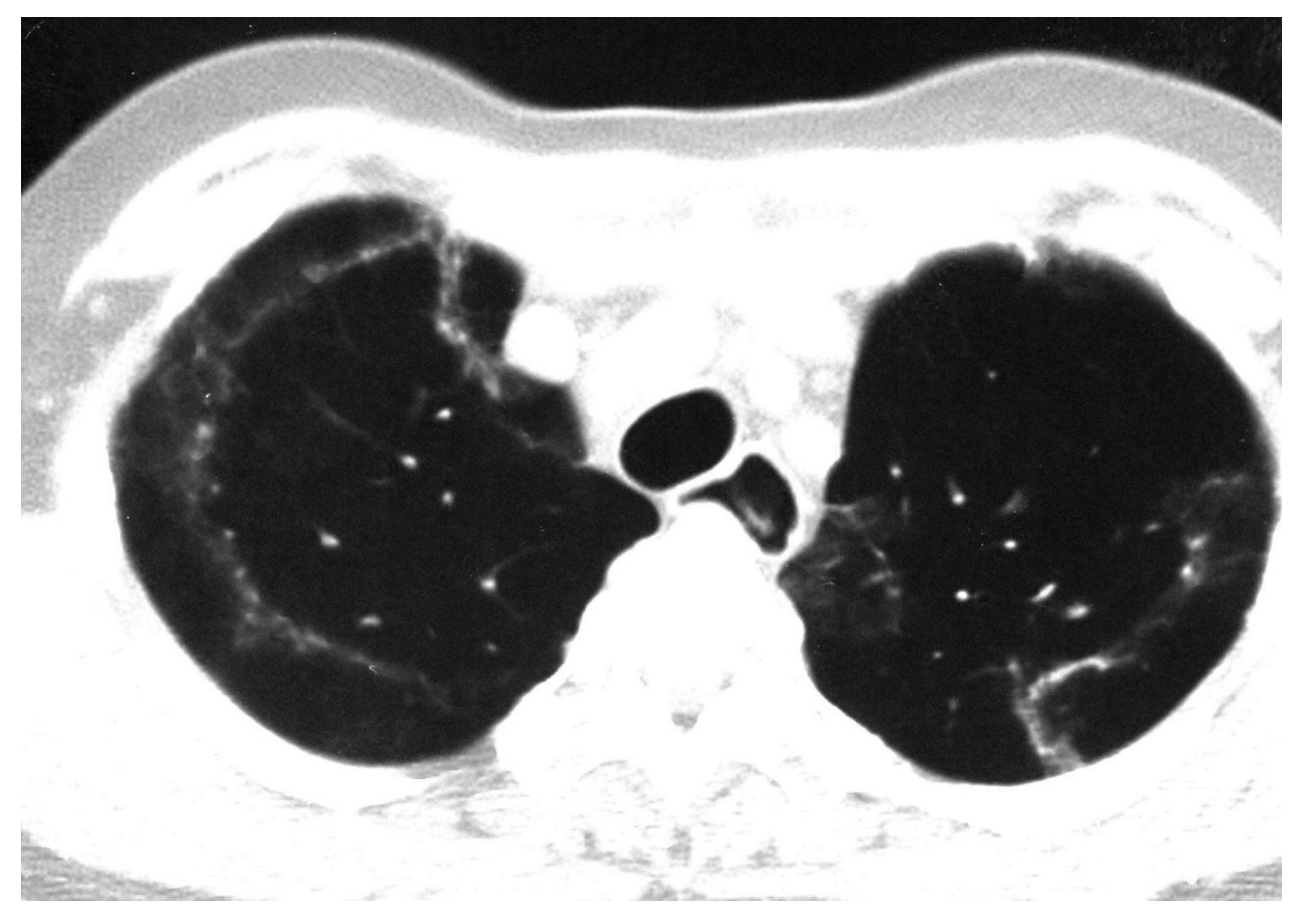

Fig. 3: CT scan on admission also revealed subpleural curvilinear shadow within both upper lobes of the lungs. 
in the bilateral upper lungs (Fig. 2). In addition, SCLS was found in the bilateral upper lungs (Fig. 3). The distribution of the infiltrates was nonsegmental. All tests for acid-fast bacilli including culture were negative. Serologic and microscopic testing for fungi was negative. Cytoplasmic and perinuclear antineutrophil antibodies were unremarkable. Serum specific IgG against Aspergillus were negative. Pulmonary function tests showed impaired gas exchange, $\mathrm{Pa}_{\mathrm{O} 2}$ $67.0 \mathrm{~mm} \mathrm{Hg}$, alveolar-arterial oxygen gradient $\left(\mathrm{AaD}_{\mathrm{O} 2}\right) 36$ $\mathrm{mm} \mathrm{Hg}$, single breath carbon monoxide diffusing capacity $\left(\mathrm{DL}_{\mathrm{CO}}\right) 11.7 \mathrm{ml} / \mathrm{min} / \mathrm{mm} \mathrm{Hg}(70.5 \%)$, a reduced vital capacity of 2.62 liters (106.0\%), a total lung capacity (TLC) of 4.46 liters $(112.1 \%)$ and a forced expiratory volume in $1 \mathrm{~s}$ $\left(\mathrm{FEV}_{1}\right)$ of 1.8 liters $(88.7 \%)$. A bronchoalveolar lavage obtained from the right middle lobe showed $55 \%$ macrophages, $13 \%$ lymphocytes, $7 \%$ neutrophils and $25 \%$ eosinophils; no pathogenic organism was found. The transbronchial biopsy from the lesion of SCLS in the right upper lobe of the lung showed focal areas of alveolar septal thickening and marked infiltration of eosinophils. Taken together, the diagnosis of CEP was confirmed. The patient was started on $40 \mathrm{mg}$ prednisolone per day. During the next $24 \mathrm{~h}$ he improved dramatically. After 2 weeks of treatment all laboratory tests and pulmonary infiltrates had normalized. She was successfully weaned off the prednisolone over a period of five months.

\section{Discussion}

CEP is an idiopathic condition characterized by chronic and progressive clinical features and specific pathologic findings (3). The clinical manifestation is usually insidious, and the patient experiences symptoms for an average of several months before the diagnosis is made (10). Women are more frequently affected than men (3). Most patients are middle aged, and approximately $50 \%$ have asthma (7). Pulmonary function tests can be normal in mild cases but usually show restrictive defects (10). Although peripheral blood eosinophilia is usually mild or moderate but occasionally is severe (7), increased serum IgE levels are seen in two-thirds of patients (16). Pulmonary infiltrates with eosinophils is the most important finding in this disease $(3,4$, $5,6,9,17,21,22)$. Histologic examination typically shows accumulation of eosinophils and lymphocytes in the alveoli and interstitium, with interstitial fibrosis $(3,10,15)$. Our patient was middle aged woman with a 4-month history of non-productive cough, fever, and night sweats, although she had no medical history of bronchial asthma. Peripheral blood eosinophilia was mild, but the percentage of eosinophils in the BAL fluid was very high and accumulation of eosinophils in the alveoli was observed in histologic examination.

It is generally accepted that the typical chest radiographic finding in CEP is nonsegmental peripheral airspace consolidation, photographic negative shadow of pulmonary edema, involving mainly the upper lobes $(3,6,8,14)$. On chest radiograph, Jederlinic et al reported that this finding may be seen in less than $50 \%$ of cases (10). However, CT clearly demonstrates typical nonsegmental areas of airspace consolidation with peripheral predominance $(6,13)$. Pleural effusion is observed in less than $10 \%$ of cases $(3$, $10,20)$. On the other hand, SCLS, which has originally been visible in the lower dorsal lung field on chest CT scan in patients with pulmonary fibrosis and asbestosis $(1,12$, $24)$, is very rare in patients with CEP $(6,18,19)$. In 1994 , Ebara et al evaluated CT features of 17 CEP patients (6). They described that linear band-like opacities parallel to the pleural surface was observed in a CEP patient on CT scan (6). Thereafter, Jeong referred the article by Ebara et al (6), and they reported that the linear band-like opacities parallel to the pleural surface predominated in the later stages of CEP (11). In the original report by Ebara et al, however, only one patient showed SCLS on CT scan performed more than 2 months after onset of symptoms (6). To our knowledge, there were two additional CEP patients with SCLS $(10,19)$. One patient had had 2 months' history of fever, chills, productive cough, and weight loss (18), but the other had only one month's history of a continuing cough, sputum, and wheezing (19). Webb et al described that SCLS in the lower dorsal lung field might be chronic changes such as fibrosis, and it might somewhat be attributed to the effect of gravity (23). Arai et al showed two cases of transient SCLS caused by pulmonary congestion, and they speculated that SCLS was not a specific finding in pulmonary fibrosis and asbestosis (2). Kashiwabara recently reported SCLS in 14 patients at hilar and heart levels on herical CT scan disappeared on prone image (12). SCLS in our patient located lateral to anterior parts of both upper lobe of the lungs. Therefore, it might not be the gravity-related chronic parenchymal abnormality. Histopathological findings of SCLS in our patient were alveolar septal thickening and marked infiltration of eosinophils. There was no fibrosis and organizing change. In addition to this, the shadow responded dramatically and was disappeared soon after the corticosteroid therapy. This response implied that the shadow might be transient change such as plate-like atelectasis or inadequate lymphatic flow from an exudative lesion.

It is possible that patients with CEP can be accompanied with SCLS. For these rare patients, therefore, we would consider performing BAL to establish correct diagnosis of CEP.

\section{References}

1. Akira M, Yamamoto S, Inoue Y, Sakatani M. High-resolution CT of asbestosis and idiopathic pulmonary fibrosis. AJR Am J Roentgenol 2003; 181: 163-9.

2. Arai K, Takashima T, Matsui O, Kadoya M, Kamimura R. Transient subpleural curvilinear shadow caused by pulmonary congestion. J Comput Assist Tomogr 1990; 14: 87-8.

3. Carrington CB, Addington WW, Goff AM, et al. CEP. N Engl J Med 1969; 280: 787-98.

4. Chapman BJ, Capewell R, Gibson R, Greening AP, Crompton GK. Pulmonary eosinophilia with and without allergic bronchopulmonary aspergillosis. Thorax 1989; 44: 919-24. 
5. Dejaegher P, Demedts M. Bronchoalveolar lavage in eosinophilic pneumonia before and during corticosteroid therapy. Am Rev Respir Dis 1984; 129: 631-2.

6. Ebara H, Ikezoe J, Johkoh T, et al.CEP: Evolution of chest radiograms and CT features. J Comput Assist Tomogr 1994; 18: 737-44

7. Fox B, Seed WA. CEP. Thorax 1980; 35: 570-580.

8. Gaensler EA, Carrington CB. Peripheral opacities in CEP: the photographic negative of pulmonary edema. AJR Am J Roentgenol 1977; 128: 1-13.

9. Janin A, Torpier G, Courtin P, et al. Segregation of eosinophil proteins in alveola macrophage compartments in CEP. Thorax 1993; 48: 57-62.

10. Jederlinic PJ, Sicilian L, Gaensler EA. CEP: a report of 19 cases and a review of the literature. Medicine 1988; 67: 154-62.

11. Jeong YJ, Kim KI, Seo IJ, et al. Eosinophilic lung diseases: a clinical, radiologic, and pathologic overview. Radiographics 2007; 27: 617-37.

12. Kashiwabara K. Characteristics and disease activity of early interstitial lung disease in subjects with true parenchymal abnormalities in the posterior subpleural aspect of the lung. Chest 2006; 129: 402-6.

13. Mayo JR, Muller NL, Road J, Sisler J, Lillington G. CEP: CT findings in six cases. AJR Am J Roentgenol 1989; 153: 727-30

14. McCarthy DS, Pepys J. Cryptogenic pulmonary eosinophilias. Clin Allergy 1973; 3. 339-51.

15. Mochimaru H, Kawamoto M, Fukuda Y, Kudoh S. Clinicopathological differences between acute and CEP. Respirology 2005; 10: 76-85.
16. Naughton M, Fahy J, FitzGerald MX. CEP: a long-term follow-up of 12 patients Chest 1993; 103: 162-5

17. Naughton M, Fahy J, Fitzgerald MX. CEP. A long-term followup of 12 patients. Chest 1993; 103: 162-5.

18. Onitsuka H, Onitsuka S, Yokomizo Y, Matsuura K. Computed tomography of chronic eosinophilic pneumonia. J Comput Assist Tomogr 1983; 7: 1092-4.

19. Saito S, Tsuneta Y, Nishiura Y, Takahashi W, Kato H, Kawai T. A case of chronic eosinophilic pneumonia with a subpleural curvilinear shadow. Nihon Kyobu Shikkan Gakkai Zasshi 1995; 33: 528-32 (in Japanese with English abstract.

20. Samman YS, Wali SO, Abdelaal MA, Gangi MT, Krayem AB. CEP presenting with recurrent massive bilateral pleural effusion. Chest 2001; 119: 968-70.

21. Takahashi H, Arakawa Y, Oki K, Sugita H, Sakamoto S, Kawabatay S. Analysis of bronchoalveolar lavage cells in CEP before and during corticosteroid therapy. Int Arch Allergy Immunol 1995; 108(suppl 1): 2-5.

22. Umeki U. Reevaluation of eosinophilic pneumonia and its diagnostic criteria. Arch Intern Med 1992; 152: 1913-9.

23. Webb WR, Muller NL, Naidich DP. High-resolution CT of the lung. $3^{\text {rd }}$ ed. Philadelphia, PA: Lppincott Williams \& Wilkins, 2001: 14-22.

24. Yoshimura $\mathrm{H}$, Hatakeyama M, Otsuji $\mathrm{H}$, et al. Pulmonary asbestosis: $\mathrm{CT}$ study of subpleural curvilinear shadow. Work in progress. Radiology 1986; 158: 653-8.

Received: $16 / 04 / 2010$

Accepted: 10/12/2010.

\section{Corresponding author:}

Hiroaki Satoh, MD, Department of Internal Medicine, Mito Medical Center, University of Tsukuba, Mito, Ibaraki, 310-0015, Japan; e-mail: hirosato@md.tsukuba.ac.jp 